QUARTERLY OF APPLIED MATHEMATICS

VOLUME LXIX, NUMBER 3

SEPTEMBER 2011, PAGES 465-475

S 0033-569X(2011)01259-1

Article electronically published on April 25, 2011

\title{
GLOBAL ATTRACTORS FOR THE SUSPENSION BRIDGE EQUATIONS WITH NONLINEAR DAMPING
}

\author{
BY \\ JONG-YEOUL PARK (Department of Mathematics, Pusan National University, Busan 609-735, \\ Korea) \\ AND \\ JUM-RAN KANG (Department of Mathematics, Dong-A University, Busan 604-714, Korea)
}

Abstract. In this paper, we prove the existence of a global attractor for the suspension bridge equations with nonlinear damping.

1. Introduction. In this paper, we consider the asymptotic behavior of the solutions of the following initial-boundary value problem:

$$
\left\{\begin{array}{l}
u_{t t}+\Delta^{2} u+k u^{+}+a(x) g\left(u_{t}\right)+f(u)=h(x), \text { in } \Omega \times \mathbb{R}^{+}, \\
u=\Delta u=0, \text { on } \Gamma, \\
u(\tau, x)=u_{0}(x), u_{t}(\tau, x)=u_{1}(x), \text { in } \Omega,
\end{array}\right.
$$

where $\Omega$ is a bounded domain of $\mathbb{R}^{2}$ with a smooth boundary $\Gamma$ and $\tau \in \mathbb{R}^{+} . u(x, t)$ is an unknown function, which represents the deflection of the road bed in the vertical plane. $k$ denotes the spring constant and $h(x) \in L^{2}(\Omega)$. The function

$$
u^{+}=\left\{\begin{array}{l}
u, \text { if } u>0 \\
0, \text { if } u \leq 0
\end{array}\right.
$$

The function $a(x)$ satisfies:

$$
a(x) \in L^{\infty}(\Omega), a(x) \geq \alpha_{0}>0 \text { in } \Omega,
$$

where $\alpha_{0}$ is a constant.

Received November 25, 2009.

2010 Mathematics Subject Classification. Primary 35B40, 35B41, 35Q35.

Key words and phrases. Suspension bridge equations, Global attractor, Nonlinear damping.

The second author is the corresponding author.

E-mail address: jyepark@pusan.ac.kr

E-mail address: jrkang@donga.ac.kr

(C)2011 Brown University

Reverts to public domain 28 years from publication 
The nonlinear function $f \in C^{2}(\mathbb{R})$ satisfies the following assumptions: There exists a constant $C_{1}>0$ such that

$$
\begin{aligned}
& \liminf _{|s| \rightarrow \infty} \frac{F(s)}{s^{2}} \geq 0, \quad F(s)=\int_{0}^{s} f(\tau) d \tau, \\
& \limsup _{|s| \rightarrow \infty} \frac{\left|f^{\prime}(s)\right|}{|s|^{\gamma}}=0, \text { where } 0 \leq \gamma<\infty, \\
& \liminf _{|s| \rightarrow \infty} \frac{s f(s)-C_{1} F(s)}{s^{2}} \geq 0 .
\end{aligned}
$$

The damping function $g \in C^{1}(\mathbb{R})$ satisfies

$$
\begin{aligned}
& g(0)=0, g \text { strictly increasing, and } \liminf _{|s| \rightarrow \infty} g^{\prime}(s)>0, \\
& |g(s)| \leq C_{2}\left(1+|s|^{q}\right),
\end{aligned}
$$

with $1 \leq q<\infty$.

The suspension bridge equations were presented by Lazer and McKenna as new problems in the field of nonlinear analysis [9]. For the problem corresponding to (1.1) without nonlinear damping $a(x) g\left(u_{t}\right)$, there are many classical results. We refer the reader to [1], 2], 9], 10], [11, 12], [13, [14, [16] and the references therein. An 2] obtained the existence and uniqueness of a weak solution for $k>-1$ and then showed decay estimates of the solution for the suspension problem. Ma and Zhong [10] investigated the existence of global attractors of the coupled system of a suspension bridge in the space $H_{0}^{2}(\Omega) \times L^{2}(\Omega)$. Zhong et al. [16] showed the existence of strong solutions and global attractors for the suspension bridge equations in the stronger space.

The long-time behavior of solutions for the equation with nonlinear damping has attracted much attention in recent years; we refer the reader to [3, [4, [5], 6], 7], 8], [15. Chueshov and Lasiecka [4] studied the existence of weak attractors for von Karman equations with nonlinear dissipation. In that article the authors have proved the existence of a global attractor for large values of the damping parameter. Khanmamedov [5], 6] proved the global attractors for von Karman equations with nonlinear interior dissipation. Recently, Yang and Zhong [15] studied the existence of a global attractor for the plate equation without assuming large values for the damping parameter.

Motivated by the work in [16], we study the existence of the global attractor for suspension bridge equations with nonlinear damping. We use the methods provided by Yang and Zhong [15] to show the existence of the global attractor.

With the usual notation, we introduce the spaces $H=L^{2}(\Omega), V=H_{0}^{2}(\Omega)$, and endow these spaces with the usual scalar products and norms, $(\cdot, \cdot),|\cdot|,((\cdot, \cdot)),\|\cdot\|$, where

$$
(u, v)=\int_{\Omega} u(x) v(x) d x, \quad((u, v))=\int_{\Omega} \Delta u(x) \Delta v(x) d x .
$$

From the Poincaré inequality, there exists a proper constant $C_{\lambda}>0$ such that

$$
\|u\|^{2} \geq C_{\lambda}|u|^{2}, \forall u \in V .
$$

The notation used in this paper is standard. The organization of this paper is as follows. In Section 2, we give some notation and prove some lemmas in order to show 
asymptotic compactness of $S(t)$. In Section 3, we establish the existence of a bounded absorbing set in $V \times H$. In Section 4, we prove the existence of a global attractor in $V \times H$.

2. Preliminaries and abstract results. In this section, we recall some definitions and results concerning the attractor. It is known that under conditions (1.2)-(1.7) the solution operator $S(t)=\left(u(t), u_{t}(t)\right), t \geq 0$, of problems $(1.1)$ generates a $\left(C_{0}\right)$ semigroup on the energy space $V \times H$ (see [10, [15], [16]).

TheOREm 2.1 ([10], [15]). Let $\Omega$ be a bounded domain of $\mathbb{R}^{2}$ with smooth boundary, under assumptions (1.2)-(1.7). Then for any initial data $\left(u_{0}, u_{1}\right) \in V \times H$, problems (1.1) have a unique global solution $\left(u(t), u_{t}(t)\right) \in C([0, T] ; V \times H)$ for any $T>0$, and $\left(u(t), u_{t}(t)\right)$ depends continuously on $\left(u_{0}, u_{1}\right)$.

Next, we recall the simple compactness criterion stated as [3], [4, [15].

Definition 2.1 ([4, 15]). Let $X$ be a Banach space and $B$ be a bounded subset of $X$. We call a function $\phi(\cdot, \cdot)$ which is defined on $X \times X$ a contractive function on $B \times B$ if for any sequence $\left\{x_{n}\right\}_{n=1}^{\infty} \subset B$, there is a subsequence $\left\{x_{n_{k}}\right\}_{k=1}^{\infty} \subset\left\{x_{n}\right\}_{n=1}^{\infty}$ such that

$$
\lim _{k \rightarrow \infty} \lim _{l \rightarrow \infty} \phi\left(x_{n_{k}}, x_{n_{l}}\right)=0 .
$$

Denote all such contractive functions on $B \times B$ by $C(B)$.

Theorem $2.2\left([4,[15])\right.$. Let $\{S(t)\}_{t \geq 0}$ be a semigroup on a Banach space $(X,\|\cdot\|)$ that has a bounded absorbing set $B_{0}$. Moreover, assume that for any $\epsilon \geq 0$ there exist $T=T\left(B_{0}, \epsilon\right)$ and $\phi_{T}(\cdot, \cdot) \in C(B)$ such that

$$
\|S(T) x-S(T) y\| \leq \epsilon+\phi_{T}(x, y) \text { for all } x, y \in B_{0},
$$

where $\phi_{T}$ depends on $T$. Then $\{S(t)\}_{t \geq 0}$ is asymptotically compact in $X$; i.e., for any bounded sequence $\left\{y_{n}\right\}_{n=1}^{\infty} \subset X$ and $\left\{t_{n}\right\}$ with $t_{n} \rightarrow \infty,\left\{S\left(t_{n}\right) y_{n}\right\}_{n=1}^{\infty}$ is precompact in X.

Lemma $2.1([5])$. Let $g(\cdot)$ satisfy condition (1.6). Then for any $\delta>0$ there exists $c(\delta)>0$ such that

$$
|u-v|^{2} \leq \delta+c(\delta)(g(u)-g(v))(u-v) \text { for } u, v \in \mathbb{R}
$$

3. Absorbing set in $V \times H$. In this section, we prove the existence of the bounded absorbing set in $V \times H$.

LEMmA 3.1. Under assumptions (1.2)-(1.7), the semigroups $\{S(t)\}_{t \geq 0}$ corresponding to problems (1.1) have a bounded absorbing set in $V \times H$.

Proof. We set

$$
E(t)=\frac{1}{2}\left(\left|u_{t}\right|^{2}+\|u\|^{2}+k\left|u^{+}\right|^{2}\right)+\int_{\Omega}(F(u)-h u) d x .
$$


Multiplying (1.1) by $u_{t}$ and integrating over $\Omega$, we get

$$
\frac{d}{d t} E(t)+\int_{\Omega} a(x) g\left(u_{t}\right) u_{t} d x=0
$$

so from (1.2) and (1.6) we have

$$
E(t) \leq E(0), \quad \forall t \geq 0
$$

It is obvious that (1.2) and (1.6) imply that there are $\delta>0$ and $C_{\delta}>0$ such that

$$
\begin{aligned}
& \left(a(x) g\left(u_{t}\right), u_{t}\right) \geq 2 \delta\left|u_{t}\right|^{2}-C_{\delta} \operatorname{meas}(\Omega), \\
& \left(a(x) g\left(u_{t}\right)-\delta u_{t}, u_{t}\right) \geq \delta\left|u_{t}\right|^{2}-C_{\delta} \operatorname{meas}(\Omega),
\end{aligned}
$$

and from (1.3) and (1.5) we know that there are $C_{\lambda}>C_{\lambda}^{\prime}>0$ and $C_{0}=C_{1}-2\left(C_{\lambda}^{\prime}\right)^{-1}$ such that

$$
\begin{aligned}
& (f(u), u)-C_{1} \int_{\Omega} F(u) d x \geq-\frac{C_{\lambda}^{\prime}}{4}|u|^{2}-C_{0} \operatorname{meas}(\Omega), \\
& \int_{\Omega} F(u) d x>-\frac{C_{\lambda}^{\prime}}{8}|u|^{2}-C_{0} \operatorname{meas}(\Omega),
\end{aligned}
$$

for any $u \in V$. From (1.8), (3.2) and (3.5), we obtain

$$
\begin{aligned}
-C_{1}\left(\operatorname{meas}(\Omega)+|h|^{2}\right) & \leq-C_{1}\left(\operatorname{meas}(\Omega)+|h|^{2}\right)+\frac{1}{2}\left(\left|u_{t}\right|^{2}+k\left|u^{+}\right|^{2}\right)+\frac{1}{4}\|u\|^{2} \\
& \leq E(t) \leq E(0) .
\end{aligned}
$$

So from (3.1) and (3.6), we have

$$
\int_{0}^{t} \int_{\Omega} a(x) g\left(u_{t}\right) u_{t} d x d s \leq E(0)-E(t) \leq E(0)+C_{1}\left(\operatorname{meas}(\Omega)+|h|^{2}\right), \forall t \geq 0 .
$$

By assumptions (1.6) and (1.7), we have

$$
|g(s)|^{\frac{q+1}{q}}=|g(s)|^{\frac{1}{q}} \cdot|g(s)| \leq C(1+|s|)|g(s)| \leq \begin{cases}C, & |s| \leq 1 \\ 2 C g(s) s, & |s| \geq 1\end{cases}
$$


where $C$ is a constant which is independent of $s$. Then from (1.2), (3.8), using Hölder's inequality and Young's inequality, we obtain

$$
\begin{aligned}
\mid \int_{\Omega} a( & x) g\left(u_{t}\right) u d x \mid \\
\leq & \int_{\Omega\left(\left|u_{t}\right| \leq 1\right)}\left|a(x) g\left(u_{t}\right) u\right| d x+\int_{\Omega\left(\left|u_{t}\right| \geq 1\right)}\left|a(x) g\left(u_{t}\right) u\right| d x \\
\leq & \int_{\Omega\left(\left|u_{t}\right| \leq 1\right)} g(1)|a(x) u| d x \\
& +\left(\int_{\Omega\left(\left|u_{t}\right| \geq 1\right)} a(x)\left|g\left(u_{t}\right)\right|^{\frac{q+1}{q}} d x\right)^{\frac{q}{q+1}}\left(\int_{\Omega\left(\left|u_{t}\right| \geq 1\right)} a(x)|u|^{q+1} d x\right)^{\frac{1}{q+1}} \\
\leq & \int_{\Omega\left(\left|u_{t}\right| \leq 1\right)} C|a(x) u| d x \\
& +\left(\int_{\Omega\left(\left|u_{t}\right| \geq 1\right)} 2 C a(x) g\left(u_{t}\right) u_{t} d x\right)^{\frac{q}{q+1}}\left(\int_{\Omega\left(\left|u_{t}\right| \geq 1\right)} a(x)|u|^{q+1} d x\right)^{\frac{1}{q+1}} \\
\leq & C \int_{\Omega} a(x)|u| d x+C_{\eta}|| u||^{\frac{q-1}{q}} \int_{\Omega} a(x) g\left(u_{t}\right) u_{t} d x+\eta \|\left. u\right|^{2},
\end{aligned}
$$

where $\eta$ is a constant. We set $v=u_{t}+\delta u$ and rewrite the equation of (1.1) as follows:

$$
v_{t}+\Delta^{2} u+k u^{+}-\delta u_{t}+a(x) g\left(u_{t}\right)+f(u)=h .
$$

Taking the scalar product in $L^{2}(\Omega)$ of (3.10) with $v$ and integrating over $\Omega$, where $\delta$ comes from (3.3), we obtain

$$
\begin{aligned}
& \frac{d}{d t}\left(\frac{1}{2}\left(|v|^{2}+\|u\|^{2}+k\left|u^{+}\right|^{2}-\delta^{2}|u|^{2}\right)+\int_{\Omega}(F(u)-h u) d x\right)+\delta\|u\|^{2}+\delta k\left|u^{+}\right|^{2} \\
& +\left(a(x) g\left(u_{t}\right)-\delta u_{t}, u_{t}\right)+\delta\left(a(x) g\left(u_{t}\right), u\right)+\delta(f(u), u)=\delta(h, u) .
\end{aligned}
$$

Set

$$
E_{\delta}(t)=\frac{1}{2}|v|^{2}+\frac{k}{2}\left|u^{+}\right|^{2}-\frac{\delta^{2}}{2}|u|^{2}+\frac{1}{2}\|u\|^{2}+\int_{\Omega}(F(u)-h u) d x
$$

and

$$
\begin{aligned}
H(t)= & \left(a(x) g\left(u_{t}\right)-\delta u_{t}, u_{t}\right)+\delta\left(a(x) g\left(u_{t}\right), u\right)+\delta\|u\|^{2}+\delta k\left|u^{+}\right|^{2} \\
& +\delta(f(u), u)-\delta(h, u),
\end{aligned}
$$

so we have

$$
\frac{d}{d t} E_{\delta}(t)+H(t)=0
$$

Now, using (3.5) and Young's inequality, we can choose $\delta$ small enough such that

$$
E_{\delta}(t) \geq \frac{1}{2}|v|^{2}+\frac{k}{2}\left|u^{+}\right|^{2}+\frac{1}{4}\|u\|^{2}-C_{1}\left(\operatorname{meas}(\Omega)+|h|^{2}\right) .
$$


Similarly, from (1.8), (3.3)-(3.5) and (3.9), we get

$$
\begin{aligned}
H(t) \geq & \delta\left|u_{t}\right|^{2}-C_{\delta} \operatorname{meas}(\Omega)+\delta k\left|u^{+}\right|^{2}+\delta\|u\|^{2} \\
& -\delta\left(C \int_{\Omega} a(x)|u| d x+C_{\eta}\left\|\left.u\right|^{\frac{q-1}{q}} \int_{\Omega} a(x) g\left(u_{t}\right) u_{t} d x+\eta\right\| u \|^{2}\right) \\
& -\delta\left(\frac{C_{1}}{2}+1\right) \frac{C_{\lambda}^{\prime}}{4}|u|^{2}-\delta C_{0} \operatorname{meas}(\Omega)\left(C_{1}+1\right)-\frac{\delta C_{\lambda}^{\prime}}{4}|u|^{2}-\frac{\delta}{C_{\lambda}^{\prime}}|h|^{2} \\
\geq & \delta\left|u_{t}\right|^{2}-C_{\delta}^{\prime}\left(\operatorname{meas}(\Omega)+|h|^{2}\right)+\delta k\left|u^{+}\right|^{2}+\delta\left(\frac{1}{2}-\frac{C_{1}}{8}-\eta\right)\|u\|^{2} \\
& -\delta\left(C \int_{\Omega} a(x)|u| d x+C_{\eta}|| u \|^{\frac{q-1}{q}} \int_{\Omega} a(x) g\left(u_{t}\right) u_{t} d x\right) \\
\geq & C_{\delta}^{\prime \prime}\left(\left|u_{t}\right|^{2}+k\left|u^{+}\right|^{2}+\|u\|^{2}\right)-C_{\delta}^{\prime}\left(\operatorname{meas}(\Omega)+|h|^{2}\right) \\
& -C_{E(0)} a(x) g\left(u_{t}\right) u_{t} d x,
\end{aligned}
$$

where we can choose $\eta$ so small that $\frac{1}{2}-\frac{C_{1}}{8}-\eta>0 ; C_{E(0)}$ is a constant which depends on $\delta, C_{\eta}$ and $E(0) ; C_{\delta}^{\prime \prime}$ and $C_{\delta}^{\prime}$ are constants depending on $\delta$ and $C_{1}$. Note that

$$
\begin{aligned}
\left|u_{t}\right|^{2}+\|u\|^{2} & =\left|u_{t}+\delta u-\delta u\right|^{2}+\|u\|^{2} \\
& \leq 2|v|^{2}+\left(\frac{2 \delta^{2}}{C_{\lambda}}+1\right)\|u\|^{2} \leq c_{0}\left(|v|^{2}+\|u\|^{2}\right),
\end{aligned}
$$

where $c_{0}=\max \left\{2,1+2 \delta^{2} C_{\lambda}^{-1}\right\}$. Integrating (3.11), combining with (3.7), (3.12)-(3.14), we deduce

$$
\begin{aligned}
& \left|u_{t}\right|^{2}+k\left|u^{+}\right|^{2}+\|u\|^{2}-2 c_{0} C_{1}\left(\operatorname{meas}(\Omega)+|h|^{2}\right)-2 c_{0} E_{\delta}(0) \\
& -2 c_{0} C_{E(0)}\left(E(0)+C_{1}\left(\operatorname{meas}(\Omega)+|h|^{2}\right)\right) \\
\leq & -2 c_{0} \int_{0}^{t}\left(C_{\delta}^{\prime \prime}\left(\left|u_{t}(s)\right|^{2}+k\left|u^{+}(s)\right|^{2}+|| u(s) \|^{2}\right)-C_{\delta}^{\prime}\left(\operatorname{meas}(\Omega)+|h|^{2}\right)\right) d s .
\end{aligned}
$$

Therefore, for any $\rho>\frac{C_{\delta}^{\prime}\left(\operatorname{meas}(\Omega)+|h|^{2}\right)}{C_{\delta}^{\prime \prime}}$, there exists $t_{0}$ such that

$$
\left.|| u\left(t_{0}\right)\right|^{2}+\left|u_{t}\left(t_{0}\right)\right|^{2}+k\left|u^{+}\left(t_{0}\right)\right|^{2} \leq \rho .
$$

Set

$$
B_{0}=\left\{\left(u_{0}, v_{0}\right) \in V \times\left. H||\left|u_{0} \|^{2}+\right| v_{0}\right|^{2}+\left|u_{0}^{+}\right|^{2} \leq \rho\right\},
$$

so we have that $B_{0}$ is a bounded absorbing set. Define

$$
B_{1}=\bigcup_{t \geq 0} S(t) B_{0}
$$

therefore, $B_{1}$ is also a bounded absorbing set.

4. Existence of global attractor in $V \times H$. In this section, we will first give some a priori estimates about the energy inequalities on account of the idea presented in $[\underline{3}$, [4], [5], 15]. Then we use Theorem 4.1 to establish the asymptotic compactness in $V \times H$. 
For convenience, we always denote by $B_{1}$ a bounded absorbing set obtained in Lemma 3.1. We will use the following notation:

$$
E_{w}(t)=\frac{1}{2}\left|w_{t}(t)\right|^{2}+\frac{k}{2}\left|w(t)^{+}\right|^{2}+\frac{1}{2}|| w(t) \|^{2} .
$$

\subsection{A priori estimates}

To obtain the asymptotic compactness, we establish a priori estimates. The following process is derived from the standard energy method given in [3], [4, [5, [15].

Let $\left(u_{i}(t), u_{i_{t}}(t)\right)(i=1,2)$ be the corresponding solution to $\left(u_{0}^{i}, v_{0}^{i}\right) \in B_{1}$ and let $w(t)=u_{1}(t)-u_{2}(t)$. Then $w(t)$ satisfies

$$
w_{t t}+a(x)\left(g\left(u_{1_{t}}\right)-g\left(u_{2_{t}}\right)\right)+\Delta^{2} w+k w^{+}+f\left(u_{1}\right)-f\left(u_{2}\right)=0,
$$

with the initial condition $\left(w(0), w_{t}(0)\right)=\left(u_{0}^{1}-u_{0}^{2}, v_{0}^{1}-v_{0}^{2}\right)$. At first, multiplying (4.1) by $w$ and integrating over $[0, T] \times \Omega$, we get

$$
\begin{aligned}
& \int_{0}^{T}|| w(s) \|^{2} d s+k \int_{0}^{T}\left|w(s)^{+}\right|^{2} d s=\int_{\Omega} w_{t}(0) w(0) d x-\int_{\Omega} w_{t}(T) w(T) d x \\
& +\int_{0}^{T}\left|w_{t}(s)\right|^{2} d s-\int_{0}^{T} \int_{\Omega}\left(f\left(u_{1}(s)\right)-f\left(u_{2}(s)\right)\right) w(s) d x d s \\
& -\int_{0}^{T} \int_{\Omega} a(x)\left(g\left(u_{1_{t}}(s)\right)-g\left(u_{2_{t}}(s)\right)\right) w(s) d x d s .
\end{aligned}
$$

Secondly, multiplying (4.1) by $w_{t}$ and integrating over $[s, T] \times \Omega$, we obtain

$$
\begin{aligned}
E_{w}(T) & +\int_{s}^{T} \int_{\Omega} a(x)\left(g\left(u_{1_{t}}(\tau)\right)-g\left(u_{2_{t}}(\tau)\right)\right) w_{t}(\tau) d x d \tau \\
\leq & E_{w}(s)-\int_{s}^{T} \int_{\Omega}\left(f\left(u_{1}(\tau)\right)-f\left(u_{2}(\tau)\right)\right) w_{t}(\tau) d x d \tau
\end{aligned}
$$

where $0 \leq s \leq T$. Integrating (4.3) over $[0, T]$ with respect to $s$, we have that

$$
T E_{w}(T) \leq \int_{0}^{T} E_{w}(s) d s-\int_{0}^{T} \int_{s}^{T} \int_{\Omega}\left(f\left(u_{1}(\tau)\right)-f\left(u_{2}(\tau)\right)\right) w_{t}(\tau) d x d \tau d s
$$

Moreover from (1.2), (4.3) and Lemma 2.1, we obtain that, for any $\delta>0$,

$$
\begin{aligned}
\int_{0}^{T}\left|w_{t}(\tau)\right|^{2} d \tau \leq & \delta T \operatorname{meas}(\Omega)+C_{2} E_{w}(0) \\
& -C_{2} \int_{0}^{T} \int_{\Omega}\left(f\left(u_{1}(\tau)\right)-f\left(u_{2}(\tau)\right)\right) w_{t}(\tau) d x d \tau
\end{aligned}
$$


where a constant $C_{2}=C_{\delta} \alpha_{0}^{-1}$. Thus, from (4.2) and (4.5) we have

$$
\begin{aligned}
\int_{0}^{T} E_{w}(s) d s \leq & \delta T \operatorname{meas}(\Omega)+C_{2} E_{w}(0)+\int_{\Omega} w_{t}(0) w(0) d x-\int_{\Omega} w_{t}(T) w(T) d x \\
& -C_{2} \int_{0}^{T} \int_{\Omega}\left(f\left(u_{1}(\tau)\right)-f\left(u_{2}(\tau)\right)\right) w_{t}(\tau) d x d \tau \\
& -\int_{0}^{T} \int_{\Omega}\left(f\left(u_{1}(s)\right)-f\left(u_{2}(s)\right)\right) w(s) d x d s \\
& -\int_{0}^{T} \int_{\Omega} a(x)\left(g\left(u_{1_{t}}(s)\right)-g\left(u_{2_{t}}(s)\right)\right) w(s) d x d s
\end{aligned}
$$

Now, we are going to estimate the last term in (4.6). Multiplying (1.1) by $u_{i_{t}}(t)$, we obtain

$$
\frac{1}{2} \int_{\Omega}\left(\left|u_{i_{t}}\right|^{2}+\left|\Delta u_{i}\right|^{2}+k\left|u_{i}^{+}\right|^{2}+2 F(u)\right) d x+\int_{\Omega} a(x) g\left(u_{i_{t}}\right) u_{i_{t}} d x=\int_{\Omega} h u_{i_{t}} d x,
$$

which, combining with the existence of a bounded absorbing set, implies that

$$
\int_{0}^{T} \int_{\Omega} a(x) g\left(u_{i_{t}}\right) u_{i_{t}} d x d s \leq C_{\rho} \quad(i=1,2)
$$

where $C_{\rho}$ is a constant which depends on meas $(\Omega),|h|^{2}$ and the size of $B_{1}$. By the similar method of (3.9) and (4.7), we obtain

$$
\begin{aligned}
\mid \int_{0}^{T} & \int_{\Omega} a(x) g\left(u_{i_{t}}(s)\right) w(s) d x d s \mid \\
\leq & \int_{0}^{T} \int_{\Omega\left(\left|u_{i_{t}}\right| \leq 1\right)}\left|a(x) g\left(u_{i_{t}}(s)\right) w(s)\right| d x d s+\int_{0}^{T} \int_{\Omega\left(\left|u_{i_{t}}\right| \geq 1\right)}\left|a(x) g\left(u_{i_{t}}(s)\right) w(s)\right| d x d s \\
\leq & C \int_{0}^{T} \int_{\Omega\left(\left|u_{i_{t}}\right| \leq 1\right)}|a(x) w| d x d s \\
& +\left(\int_{0}^{T} \int_{\Omega\left(\left|u_{i_{t}}\right| \geq 1\right)} a(x)\left|g\left(u_{i_{t}}\right)\right|^{\frac{q+1}{q}} d x d s\right)^{\frac{q}{q+1}}\left(\int_{0}^{T} \int_{\Omega\left(\left|u_{i_{t}}\right| \geq 1\right)} a(x)|w|^{q+1} d x d s\right)^{\frac{1}{q+1}} \\
\leq & C \int_{0}^{T} \int_{\Omega\left(\left|u_{i_{t}}\right| \leq 1\right)}|a(x) w| d x d s \\
& +2 C\left(C_{\rho}\right)^{\frac{1}{q+1}} T^{\frac{1}{q+1}}\left(\int_{0}^{T} \int_{\Omega\left(\left|u_{i_{t}}\right| \geq 1\right)} a(x) g\left(u_{i_{t}}\right) u_{i_{t}} d x d s\right)^{\frac{q}{q+1}} \\
\leq & C \int_{0}^{T} \int_{\Omega} a(x)|w| d x+2 C C_{\rho} T^{\frac{1}{q+1}}(i=1,2) .
\end{aligned}
$$


Combining (4.4), (4.6) and (4.8), we have

$$
\begin{aligned}
T E_{w}(T) \leq & \delta T \operatorname{meas}(\Omega)+C_{2} E_{w}(0)+\int_{\Omega} w_{t}(0) w(0) d x-\int_{\Omega} w_{t}(T) w(T) d x \\
& +C \int_{0}^{T} \int_{\Omega} a(x)|w| d x-\int_{0}^{T} \int_{\Omega}\left(f\left(u_{1}(s)\right)-f\left(u_{2}(s)\right)\right) w(s) d x d s \\
& +2 C C_{\rho} T^{\frac{1}{q+1}}-C_{2} \int_{0}^{T} \int_{\Omega}\left(f\left(u_{1}(\tau)\right)-f\left(u_{2}(\tau)\right)\right) w_{t}(\tau) d x d \tau \\
& -\int_{0}^{T} \int_{s}^{T} \int_{\Omega}\left(f\left(u_{1}(\tau)\right)-f\left(u_{2}(\tau)\right)\right) w_{t}(\tau) d x d \tau d s .
\end{aligned}
$$

Set

$$
\begin{aligned}
& C_{B}= \delta T \operatorname{meas}(\Omega)+C_{2} E_{w}(0)+\int_{\Omega} w_{t}(0) w(0) d x \\
&-\int_{\Omega} w_{t}(T) w(T) d x+2 C C_{\rho} T^{\frac{1}{q+1}}, \\
& \phi_{T}\left(\left(u_{0}^{1}, v_{0}^{1}\right),\left(u_{0}^{2}, v_{0}^{2}\right)\right) \\
&=C \int_{0}^{T} \int_{\Omega} a(x)|w| d x \\
&-\int_{0}^{T} \int_{\Omega}\left(f\left(u_{1}(s)\right)-f\left(u_{2}(s)\right)\right) w(s) d x d s \\
&-C_{2} \int_{0}^{T} \int_{\Omega}\left(f\left(u_{1}(\tau)\right)-f\left(u_{2}(\tau)\right)\right) w_{t}(\tau) d x d \tau \\
&-\int_{0}^{T} \int_{s}^{T} \int_{\Omega}\left(f\left(u_{1}(\tau)\right)-f\left(u_{2}(\tau)\right)\right) w_{t}(\tau) d x d \tau d s .
\end{aligned}
$$

Then we have

$$
E_{w}(T) \leq \frac{C_{B}}{T}+\frac{1}{T} \phi_{T}\left(\left(u_{0}^{1}, v_{0}^{1}\right),\left(u_{0}^{2}, v_{0}^{2}\right)\right) .
$$

\subsection{Asymptotic compactness}

In this subsection, following similar arguments as in [5, 7], [15, we shall prove the asymptotic compactness of the semigroup $\{S(t)\}_{t \geq 0}$ in $V \times H$, which is given in the following theorem.

TheOREM 4.1. Under assumptions (1.2)-(1.7), then the semigroup $\{S(t)\}_{t \geq 0}$ corresponding to problems (1.1) is asymptotically compact in $V \times H$.

Proof. Since the semigroup $\{S(t)\}_{t \geq 0}$ has a bounded absorbing set, for any fixed $\epsilon>0$, we can first choose $\delta \leq \frac{\epsilon}{2 \operatorname{meas}(\Omega)}$, and then let $T$ be so large that

$$
\frac{C_{B}}{T} \leq \epsilon
$$

Hence, thanks to Theorem 2.2, we only need to verify that the function $\phi_{T}(\cdot, \cdot)$ defined in (4.11) belongs to $C\left(B_{1}\right)$ for each fixed $T$. Let $\left(u_{n}, u_{t_{n}}\right)$ be the corresponding solution 
of $\left(u_{0}^{n}, v_{0}^{n}\right) \in B_{1}, n=1,2, \ldots$. Then, since $B_{1}$ is a bounded positively invariant set in $V \times H$, without loss of generality, we assume that

$$
\begin{aligned}
& u_{n} \rightarrow u \text { weakly star in } L^{\infty}\left(0, T ; H_{0}^{2}(\Omega)\right), \\
& u_{n_{t}} \rightarrow u_{t} \text { weakly star in } L^{\infty}\left(0, T ; L^{2}(\Omega)\right), \\
& u_{n} \rightarrow u \text { strongly in } L^{2}\left(0, T ; L^{2}(\Omega)\right), \\
& u_{n} \rightarrow u \text { strongly in } L^{k}\left(0, T ; L^{k}(\Omega)\right),
\end{aligned}
$$

for $k \leq 2(\gamma+1)$, where we use the compact embedding $H_{0}^{2} \hookrightarrow L^{k}$. Now, we will deal with each term corresponding to that in (4.11). At first, from (4.15), we have

$$
\lim _{n \rightarrow \infty} \lim _{m \rightarrow \infty} \int_{0}^{T} \int_{\Omega}\left(f\left(u_{n}(s)\right)-f\left(u_{m}(s)\right)\right)\left(u_{n}(s)-u_{m}(s)\right) d x d s=0 .
$$

Secondly, from (4.16) and (1.2), we obtain

$$
\lim _{n \rightarrow \infty} \lim _{m \rightarrow \infty} \int_{0}^{T} \int_{\Omega} a(x)\left|u_{n}(s)-u_{m}(s)\right| d x d s=0 .
$$

Finally, following the similar argument given in [7, we get

$$
\begin{aligned}
& \lim _{n \rightarrow \infty} \lim _{m \rightarrow \infty} \int_{0}^{T} \int_{\Omega}\left(f\left(u_{n}(s)\right)-f\left(u_{m}(s)\right)\right)\left(u_{n_{t}}(s)-u_{m_{t}}(s)\right) d x d s=0, \\
& \lim _{n \rightarrow \infty} \lim _{m \rightarrow \infty} \int_{0}^{T} \int_{s}^{T} \int_{\Omega}\left(f\left(u_{n}(\tau)\right)-f\left(u_{m}(\tau)\right)\right)\left(u_{n_{t}}(\tau)-u_{m_{t}}(\tau)\right) d x d \tau d s=0 .
\end{aligned}
$$

Hence, combining (4.17)-(4.20) we get $\phi_{T}(\cdot, \cdot) \in C\left(B_{1}\right)$ immediately.

\subsection{Existence of global attractor}

Theorem 4.2. Under assumptions (1.2)-(1.7), then problems (1.1) have a global attractor in $V \times H$, which is invariant and compact.

Proof. Lemma 3.1 and Theorem 4.1 imply the existence of a global attractor.

\section{REFERENCES}

[1] N. U. Ahmed and H. Harbi, Mathematical analysis of dynamic models of suspension bridges, SIAM J. Appl. Math. 58 (1998) 853-874. MR1616611 (99d:73050)

[2] Y. An, On the suspension bridge equations and the relevant problems, Doctoral Thesis, 2001.

[3] I. Chueshov and I. Lasiecka, Attractors for second-order evolution equations with nonlinear damping, J. Dynam. Differential Equations 16 (2004) 469-512. MR.2105786 (2005g:37149)

[4] I. Chueshov and I. Lasiecka, Long-time dynamics of von Karman semi-flows with non-linear boundary/interior damping, J. Differential Equations 233 (2007) 42-86. MR2290271 (2008a:37091)

[5] A. Kh. Khanmamedov, Global attractors for von Karman equations with nonlinear interior damping, J. Math. Anal. Appl. 318 (2006) 92-101. MR2210874 (2007b:37198)

[6] A. Kh. Khanmamedov, Finite dimensionality of the global attractors for von Karman equations with nonlinear interior dissipation, Nonlinear Anal. 66 (2007) 204-213. MR.2271647 (2007f:35209)

[7] A. Kh. Khanmamedov, Global attractors for wave equations with nonlinear interior damping and critical exponents, J. Differential Equations 230 (2006) 702-719. MR2269940 (2007i:37150)

[8] A. Kh. Khanmamedov, Global attractors for the plate equation with a localized damping and a critical exponent in an unbounded domain, J. Differential Equations 225 (2006)528-548. MR2225799 (2007a:37100) 
[9] A. C. Lazer and P. J. McKenna, Large-amplitude periodic oscillations in suspension bridge: Some new connections with nonlinear analysis, SIAM Rev. 32 (1990) 537-578. MR.1084570 (92g:73059)

[10] Q.Z. Ma and C.K. Zhong, Existence of global attractors for the coupled system of suspension bridge equations, J. Math. Anal. Appl. 308 (2005) 365-379. MR2142424(2006b:37167)

[11] Q.Z. Ma and C.K. Zhong, Existence of global attractors for the suspension bridge equations, J. Sichuan Univ. 43 (2) (2006). MR2226668

[12] P. J. McKenna and W. Walter, Nonlinear oscillation in a suspension bridge, Nonlinear Anal. 39 (2000) 731-743.

[13] J.Y. Park and J.R. Kang, Pullback D-attractors for non-autonomous suspension bridge equations, Nonlinear Anal. 71 (2009) 4618-4623. MR.2548694 (2010i:35258)

[14] R. Temam, Infinite-dimensional Dynamical Systems in Mechanics and Physics, Springer-Verlag, 1997. MR 1441312 (98b:58056)

[15] L. Yang and C.K. Zhong, Global attractor for plate equation with nonlinear damping, Nonlinear Anal. 69 (2008) 3802-3810. MR2463335 (2009k:35211)

[16] C.K. Zhong, Q.Z. Ma, and C.Y. Sun, Existence of strong solutions and global attractors for the suspension bridge equations, Nonlinear Analysis 67 (2007) 442-454. MR2317179 (2008h:35250) 